\title{
Meeting report \\ Highlights from the 37th Annual Meeting of the American Society of Clinical Oncology, San Francisco, USA, 12-15 May 2001
}

\author{
Caroline Lohrisch
}

Institute Jules Bordet, Brussels, Belgium

Correspondence: Caroline Lohrisch MD, Institute Jules Bordet, Bd de Waterloo, 125, 1000 Brussels, Belgium. Tel: +32 25413082 ; fax: +32 2538 7363; e-mail: caroline.lohrisch.idbbc@bordet.be

\begin{abstract}
Key issues discussed at the breast cancer sessions of the 37th American Society of Clinical Oncology (ASCO) meeting, 2001, included the following: breast cancer in the elderly; toxicity; updates on HER2 and use of trastuzumab (anti-HER2) in metastatic disease; and several early reports on novel therapeutic strategies. As was stated by numerous speakers both at this and other meetings, and as demonstrated by several abstracts, the use of predictive factors appears to be the most effective way to enhance the efficacy of numerous therapies, both target specific and nonspecific.
\end{abstract}

Keywords: adjuvant, endocrine, Herceptin, high-dose chemotherapy

\section{Introduction}

The goals of the ASCO annual meeting are to disseminate the latest and most worthwhile research data emerging from the fields of cancer prevention, detection, diagnosis, prognosis and therapy. The majority of these topics were covered to variable degrees in the field of breast cancer, with educational sessions focusing on current controversies and future directions, and scientific sessions filling in the gaps between our current standards and the active thrusts of research.

In the following text numbers in parentheses, in the format ' $(\# X X)$ ', indicate the number of the abstract in the proceedings book for the meeting [1].

\section{High-dose chemotherapy}

Several randomized studies of standard versus high-dose chemotherapy with stem cell support for either high-risk adjuvant or metastatic therapy were presented. Three studies in the adjuvant setting (\#80, \#81, \#148) failed to show a disease-free survival or overall survival advantage at 5 years for high-dose chemotherapy compared with standard or somewhat dose-escalated chemotherapy in women with multiple positive nodes. One trial (Pegase 01; \#102) reported better relapse-free survival for the high-dose arm than the standard dose arm ( $71 \%$ versus $55 \% ; P<0.003$ ), suggesting that longer follow-up of overall survival is required (the overall survival rates were equivalent in the two arms at 3 years follow-up). In the metastatic setting, the MA.16 study (\#82) reported equivalent overall survival for the 219 patients who were randomly assigned to 2-4 additional cycles of anthracycline- or taxane-based chemotherapy or to high-dose chemotherapy after an objective response to standard chemotherapy. In the same theme, several studies that compared standard with increased dose intensity anthracycline therapy (\#146, \#127) failed to show an advantage for the latter strategy. 


\section{HER2 and Herceptin ${ }^{\circledR}$ (trastuzumab)}

The prognostic and predictive role of HER2 continues to be a hot topic in breast cancer.

Several studies (\#85, \#86) reported that benefit from trastuzumab is confined to women with HER2 (c-erbB2) gene amplification (assessed by fluorescence in situ hybridization $[\mathrm{FISH}]$ ), independent of immunohistochemistry results $(3+$ or $2+)$. Another abstract (\#172) reported similar efficacy of trastuzumab in oestrogen receptor (ER)positive and ER-negative metastatic breast cancer (MBC).

The predictive value of HER2 for chemotherapy sensitivity was explored retrospectively in a number of randomized trials in the adjuvant and metastatic settings. An Italian group reported improved overall survival for HER2-positive patients (assessed by immunohistochemistry) who received anthracycline-based chemotherapy versus cyclophosphamide, methotrexate, 5-fluorouracil (CMF)-based chemotherapy, whereas for HER2-negative patients overall survival was equivalent for both regimens (\#89). Another Italian trial reported a trend toward improved overall survival for anthracycline over CMF therapy for women with HER2-positive disease (hazard ratio 0.85, $95 \%$ confidence interval $[\mathrm{Cl}]$ 0.27-2.71) but not for those with HER2-negative disease (hazard ratio $1.64,95 \% \mathrm{Cl}$ 0.85-3.14) (\#133). The Cls crossed 1 in both groups, however, suggesting equivalence.

In MBC, a trial that compared first-line epirubicin + paclitaxel with epirubicin + cyclophosphamide (\#88) showed equivalence for the two regimens among HER2-negative (by FISH) patients and superior overall survival for epirubicin + paclitaxel among HER2-positive patients $(P=0.06)$. Another retrospective study (\#181), however, failed to demonstrate HER2 over-expression as a predictive factor for response to taxanes. Serum HER2 level was examined as a predictive marker of response to endocrine therapy (\#87). Among 153 women, the relative risk $(P=0.0005)$, time to progression (TTP; $P<0.0001)$ and overall survival $(P<0.0001)$ were lower in the group of 51 women (33\%) with elevated levels of HER2, and for this group there was no significant difference for megace and letrozole. In the group without elevated HER2 levels, both TTP $(P=0.017)$ and overall survival $(P=0.025)$ were better for letrozole. Disease and patient characteristics, which might also have influenced TTP and overall survival, were not provided.

Other inhibitors of the HER family are in various stages of development. Iressa ${ }^{\circledR}$ (ZD1839; AstraZeneca, Alderley Park, Cheshire, UK), an epidermal growth factor (EGF) receptor tyrosine kinase inhibitor, was explored in HER2over-expressing breast cancer cell lines (\#8). Iressa ${ }^{\circledR}$ almost entirely inhibited the phosphorylation (activation) of HER2 at low concentrations. The degree of growth inhibi- tion was greater with Iressa $^{\circledR}$ than with Herceptin ${ }^{\circledR}$ (trastuzumab; Genentech Inc, San Francisco, CA, USA) and at high concentrations was able to inhibit growth in Herceptin ${ }^{\circledR}$-resistant cell lines. When given together, Herceptin $^{\circledR}$ and Iressa ${ }^{\circledR}$ were observed to induce apoptosis. Another abstract (\#282) reported inhibition of ERK1/2 activation (downstream of EGF receptor and c-erbB2) by Iressa ${ }^{\circledR}$ in tamoxifen-resistant cells, which are known to overexpress both EGF receptor and c-erbB2. Coupled with this was a marked growth inhibitory effect, which was not observed in wild-type MCF-7 cells (which are tamoxifen sensitive and do not have EGF receptor or c-erbB2 over-expression). Exploration of this drug with second-line hormone therapy is needed to determine whether in vivo effects parallel these promising findings.

\section{Endocrine therapy Adjuvant}

A randomized trial of observation versus oophorectomy plus tamoxifen in 709 premenopausal Vietnamese and Chinese women (\#99) reported substantial improvements in disease-free survival $(75 \%$ versus $58 \% ; P=0.006)$ and overall survival $(78 \%$ versus $70 \% ; P=0.04 ; 80 \%$ versus $58 \%$ among ER-positive patients) for the endocrine therapy. A German study of CMF versus a luteinizing hormone-releasing hormone analogue for 2 years in premenopausal women with node-positive breast cancer (\#132) reported no difference in progression-free survival. With only 2 years of follow-up, however, these data are immature. Quality of life data from the Zebra trial (\#138) suggested better overall tolerance during the treatment period for Zoladex ${ }^{\circledR}$ (goserelin; AstraZeneca) versus CMF

An ongoing question is the value of ovarian suppression added to chemotherapy in premenopausal women; the 2000 Oxford overview (Meeting of the Early Breast Cancer Trialists' Collaborative Group, Oxford, UK, September 2000; unpublished data) suggests that there is none. Subgroup analysis of the Zipp trial (\#103), in which 2170 women were randomly assigned to receive goserelin, tamoxifen or placebo, and could receive chemotherapy at the investigator's discretion, supported this finding. Among women with ER-positive disease, the relative risk for event-free survival of goserelin versus placebo was 0.74 for the group who had chemotherapy, and 0.86 among those with no chemotherapy, with confidence intervals crossing 1 in both analyses. In contrast, an Italian trial (\#104) that randomly assigned women to two years of goserelin plus tamoxifen versus no endocrine therapy following chemotherapy reported a hazard ratio for relapse of 0.57 for patients with ER-positive or unknown disease who received the endocrine therapy $(P=0.02)$, although no survival advantage has yet emerged (hazard ratio 0.86 ; $P=0.52$ ). In the small premenopausal subgroup of the SWOG 7827 trial (\#105), the addition of oophorectomy to one year of CMF plus vincristine and predinsone did not 
significantly alter the overall survival $(P=0.67)$. However, $100 \%$ of CMF plus vincristine and predinsone patients developed amenorrhoea during chemotherapy $(70 \%$ of those younger than 40 years recovered menses).

The reverse question has also been asked in the postmenopausal setting - does chemotherapy provide additional benefit over tamoxifen? The intergroup 0100 study (\#94), updated with 8 years of follow-up, continues to show an advantage for cyclophosphamide, doxorubicin, 5fluorouracil with tamoxifen versus tamoxifen alone in terms of disease-free survival (76\% versus $67 \% ; P=0.0002)$ and overall survival ( $84 \%$ versus $79 \% ; P=0.006)$.

\section{Metastatic}

Only a few studies of hormone therapy for MBC were reported at this year's ASCO meeting. Idoxifene, which has fewer oestrogen agonist properties than tamoxifen, was equivalent to tamoxifen in terms of response rate (RR), clinical benefit, TTP, overall survival and adverse events among postmenopausal women (\#113). Exemestane was associated with a high RR $(41 \%)$, median duration of response and of clinical benefit of 18.4 and 16.6 months, respectively, and no adverse impact on serum lipids when given as first-line therapy in a similar patient population (\#114, \#167). The activity of the selective oestrogen receptor modulator Arzoxifene ${ }^{\circledR}$ (LY-353381; Eli Lilly \& Co, Indianapolis, IN, USA) was low in tamoxifen sensitive (RR 16\%, clinical benefit 33\% TTP 5.5 months) and refractory patients (RR 7\%, clinical benefit 12\%, TTP 2.7 months; \#178).

\section{Novel therapeutic strategies for metastatic breast cancer}

A myriad of abstracts explored innovative treatment approaches, including targeted therapies, new combinations, and better patient selection based on predictive factors.

A $96 \%$ positive predictive value and $94 \%$ sensitivity were reported for 2-fluoro-2-deoxyglucose positron emission tomography scanning among 119 patients with isolated elevations in the tumour marker Ca15.3 (\#165). Among 13 negative cases, seven were false negatives and six were true negatives. This suggests that, where available, positron emission tomography scanning may be a useful tool for identifying occult disease.

Caelyx ${ }^{\circledR}$ (pegylated liposomal doxorubicin; ScheringPlough, Levallois-Perret, France) was compared with weekly vinorelbine or mitomycin plus vinblastine after taxane failure in $301 \mathrm{MBC}$ patients (\#115). The RR, progression-free survival (2.9 versus 2.5 months; $P=0.11$ ), and median overall survival (10.4 versus 9.4 months; $P=0.57$ ) were equivalent. The proportion with prior anthracycline therapy, which may have influenced response to Caelyx ${ }^{\circledR}$, was not given. Another group reported enhanced efficacy of Caelyx ${ }^{\circledR}$ with local hyperthermia given for chest wall lesions, with a local RR of $60 \%$ among 20 evaluable patients (\#184).

The antiangiogenic effects of chronic low dose oral methotrexate and cyclophosphamide were explored (\#116); 52 out of 62 enrolled patients had prior chemotherapy for MBC, and 20 had had at least two prior regimens. The overall RR was $32 \%$, but the progression-free survival was a disappointing 2.8 months. A randomized comparison of anthracycline alone or in combination with $\mathrm{N}, \mathrm{N}$-diethyl2(4-phenylmethyl-phenoxyethamine) hydrochloride (DDPE) (\#118), which in vitro enhances response to anthracycline, reported equivalent RR and TTP $(P=0.3)$ but improved overall survival $(P=0.02)$ for the combination among 305 randomized patients. Potential explanations for these results include chance, an imbalance in subsequent therapies or prognostic factors, or a sensitizing effect of DDPE to subsequent chemotherapy.

A phase I study (\#170) reported no maximum tolerated dose (up to $1000 \mathrm{mg} /$ day) and disease stabilization in 10 out of 14 evaluable patients treated with 2-methoxyoestradiol, an oestradiol metabolite that directly inhibits proliferating cells and has antiangiogenic activity. These early results are promising and accrual is ongoing. Another potentially active drug is Alimta $^{\circledR}$ (LY231514, multi-targeted antifolate; Ely Lilly \& Co), an antifolate; administration of this agent resulted in a 10\% RR and 38\% stable disease rate lasting 2.9-14.5 months (median 4.5 months) among 42 pretreated MBC patients (\#194). In contrast, a phase II trial of the anti-protein kinase C oligonucleotide ISIS 3521 (\#171) reported disappointing results, with a median TTP of 1.3 months and only one out of 15 patients free from progression at 4 months. Similarly disappointing results were observed with the matrix metalloproteinase inhibitor Prinomastat ${ }^{\circledR}$ (Pfizer, LA Jolla, CA, USA; 0\% RR, TTP 8 weeks) and oral UFT plus leucovorin following treatment with anthracyclines, taxanes, or both (11\% RR, TTP 72 days; \#188, \#186).

An oral vinorelbine formulation was examined as first-line chemotherapy in 65 patients with locally advanced or MBC at a dose of $60 \mathrm{mg} / \mathrm{m}^{2}$ per week, escalated to $80 \mathrm{mg} / \mathrm{m}^{2}$ per week if there was no significant neutropenia (\#185). Thus far, the RR by intention to treat is 31\%; TTP was not reported. Grade 3 toxicities included nausea $(6 \%)$, vomiting $(8 \%)$, diarrhoea $(6 \%)$ and grade 4 neutropenia (14\%).

Other novel therapies in the pipeline include epothilone, a microtubulin stabilizer that is active in cell lines that express multidrug resistant phenotypes (\#269, \#270); the EGF receptor-selective tyrosine kinase inhibitor ZD1839 for treatment of tamoxifen-resistant disease (\#282); 
$\mathrm{R} 115777$, a farnesyl transferase inhibitor, as a single agent and in combination with cytotoxic drugs and trastuzumab (\#322); the next generation bisphosphonate ibandronic acid (\#332); and numerous other specific inhibitors of the EGF signalling pathways, cell-cycle regulators, angiogenic mechanisms and drug-resistance mechanisms.

\section{Conclusion}

The majority of breast cancer abstracts at this year's ASCO meeting were clinical and therapeutic. Several key points summarize the focus of breast cancer research at this year's meeting.

Although not reviewed here, there was much focus on the toxicity of adjuvant therapy. Randomized trials of highdose chemotherapy confirm a lack of advantage for this modality over standard therapy in high-risk early or metastatic settings. The question of the value of adding ovarian ablation/suppression to chemotherapy in premenopausal women remains unsettled. More retrospective evidence points to a particular benefit from anthracyclines in HER2-overexpressing tumours, and to benefit from trastuzumab only in patients whose tumours have HER2 gene amplification by FISH, suggesting that FISH confirmation should be obtained when considering trastuzumab, particularly if the immunohistochemistry score is $2+$. Iressa ${ }^{\circledR}$, a EGF receptor tyrosine kinase receptor, had favourable preliminary results in inhibiting growth of HER2-overexpressing breast cancer cell lines. The use of traditional cytotoxic drugs in different formulations or dosing schedules met with variable success, and phase $\mathrm{I} / \mathrm{Il}$ results for molecules such as protein kinase $\mathrm{C}$ oligonucleotide and matrix metalloproteinases were on the whole discouraging. It is ultimately by better defining 'sensitive' patient populations, through the use of predictive markers, that we will make best use of our available and up and coming cytotoxic and target-specific therapies.

\section{Reference}

1. Grunberg SM (ed): Program Proceedings, American Society of Clinical Oncology 37th Annual Meeting Part 1 of 2. San Francisco, CA, May 12-15, 2001. Baltimore, MD: Lippincott Williams \& Wilkins; 2001 